\title{
The Injustice of Being Judged by the Errors of Others: The Tragic Tale of the Battle for PET Reimbursement
}

\author{
Rodney J. Hicks, FRACP, FAHMS, FICIS \\ Cancer Imaging, The Peter MacCallum Cancer Centre, The Sir Peter MacCallum Department of Oncology, the University of \\ Melbourne, Melbourne, Victoria, Australia
}

See the associated articles on pages 421,427 , and 434.

F or clinicians at least, it is a self-evident truth that accurate diagnosis is beneficial to patients. By providing reassurance of the absence of disease, avoiding unnecessary further investigation or futile treatments, or by detecting and characterizing disease, thereby enabling management decisions to be formulated, accurate diagnosis has formed the cornerstone of medical practice. As medical students, we are introduced to the importance of taking a careful history and performing a detailed physical examination using all our senses and a range of specialized equipment. From these observations, we are taught to formulate differential diagnoses. At times, the diagnosis is clear without further investigation and management can be determined without recourse to further investigations. At others, the diagnosis may be clear but further information is required to decide between various treatment options. More often, a definitive diagnosis cannot be reached without further investigations, which might include blood tests, examination of pathologic specimens, or imaging tests. In the modern era, genomic analysis has also entered into the diagnostic process. Fundamental to this cognitive exercise is a probabilistic integration of information until a sufficient level of certainty exists to provide advice to a patient on management options. The degree of confidence with which a diagnosis needs to be made is proportional to the consequences of misdiagnosis. When there is a risk of death or where therapeutic options involve significant morbidity or cost, a high level of diagnostic certainty is mandatory. In this context, accuracy of diagnostic paradigms becomes paramount. There can be no clearer example of the need for accurate diagnosis than when cancer is suspected. Not only is malignancy a major cause of human suffering and death, almost all the expanding array of therapies are both expensive and have a significant risk of complications (1).

Because of the importance of accurate grading and staging of cancer, pathology and imaging have long been central in the decision-making process for clinicians seeking to determine the

Received Jan. 2, 2018; revision accepted Jan. 5, 2018.

For correspondence or reprints contact: Rodney J. Hicks, Cancer Imaging, Level 5, The Victorian Comprehensive Cancer Centre, 305 Grattan St., Melbourne, VIC 3000, Australia.

E-mail: rod.hicks@petermac.org

COPYRIGHT @ 2018 by the Society of Nuclear Medicine and Molecular Imaging. DOI: 10.2967/jnumed.117.206953 best treatment options in oncology. Over the last century, there has been a marked evolution in the sophistication of these techniques and, unfortunately, often also in their cost. This has created frisson between the desires of clinicians for ever more accurate diagnosis and the financial concerns of those responsible for funding health care. Globally, this has played out in the courts of health technology assessment (HTA), with cases being prosecuted under rules codified as evidence-based medicine. Although technologies that were entrenched in clinical practice before the era of HTA have largely escaped such judgement, new technologies are now routinely considered guilty of profligate and unwarranted use by HTA agencies. In response, clinicians defend their behavior by arguing that they are best placed to balance the benefits to the patient and the cost to the community.

There can have been few more cogent examples of the cognitive dissonance between the HTA and medical communities than that pertaining to the battle for reimbursement of PET. In the late 1990s, as PET was emerging as an oncologic imaging modality, there was already a large amount of evidence that PET was significantly more accurate than conventional imaging in various malignancies (2). This was despite the relatively low technical specifications of the scanners available at that time and image quality that most nuclear medicine specialists would now consider unacceptable. The standards by which diagnostic imaging were to be judged for efficacy were, however, already being redefined to extend beyond issues of accuracy to include their impact on management and patient outcomes, including costeffectiveness (3). Responding to this environment, the nuclear medicine community began to focus on the impact of PET on patient management. One of the seminal papers in this regard came from the group of the late Peter Valk (4). Despite this accumulating evidence, HTA groups around the world remained unconvinced and access to reimbursement was severely constrained in most countries.

When I initiated our PET program in 1996, our primary goal was to establish it as a routine clinical modality. We recognized that although this would involve collection of evidence on the accuracy of PET in the detection and staging of disease, its ability to stratify prognosis, and its predictive value in assessment of response to therapies, impact on management would also need to be demonstrated. Inspired by the work of Peter Valk, we required referring clinicians to indicate, prospectively on the referral form, the planned management based on all available information up to that point in time, which almost always included conventional imaging. We then evaluated the final treatment delivered to the patient and the prognostic utility of the PET result by assessing patient outcomes. Management impact was defined as high, if 
treatment intent (curative or palliative) or modality (observation, surgery, radiotherapy, chemotherapy, or other) was changed after availability of the PET result; moderate, if delivery of a previously chosen therapy was modified; low, if PET findings suggested that the chosen management was appropriate; or of no impact, if the treatment eventually chosen was inconsistent with the findings. This last category would, for example, be applied to a patient receiving attempted curative surgery despite PET suggesting metastatic disease. We applied this approach across numerous diseases, including the staging $(5,6)$ and restaging $(7)$ of nonsmall cell lung cancer, the restaging of colorectal cancer (8), the posttreatment evaluation of head and neck cancer $(9,10)$, and the staging and posttreatment assessment of esophageal cancer $(11,12)$. These studies had validation of the appropriateness of the changes in management based on clinical follow-up and demonstrated powerful prognostic stratification based on the PET result. Many of these data were already available in late 1999 when our facility and the Wesley Hospital group in Brisbane made separate submissions to our Australia HTA group, the Medicare Services Advisory Committee (MSAC). Despite what we felt to be a compelling case based on both the international literature and our own local experience, MSAC cast doubt on the "clinical and cost-effectiveness of PET" and mandated the collection of further data. HTA agencies at that time seemed to be galvanized in delaying reimbursement of PET by this tactic, using what we and others have argued are specious arguments to deny funding of PET to the detriment of patient care (13-17). In the United States, the National Oncologic PET Registry (NOPR) was a massive logistic exercise involving thousands of patient studies. Using almost the same approach to assessment of management impact that we had used at my facility and subsequently in the Australian Data Collection PET project (18), it again confirmed the huge impact that ${ }^{18}$ F-FDG PET has on patient management across many cancer types (19). At best, this investment of human and fiscal resources in NOPR studies could be lauded for establishing the case for broadly based PET reimbursement. At worst, it could be condemned as an egregious waste of time and money proving what was already obvious and delaying what should never have been in doubt.

Three papers in the current issue of The Journal of Nuclear Medicine should add a further sad emoticon or 3 in the tragic tale of the battle fought between the PET community and HTA authorities (20-22). The papers by Hillner et al. (20) and Gareen et al. (21) were clearly motivated by an attempt to achieve reimbursement for PET with ${ }^{18} \mathrm{~F}$-fluoride for bone imaging, for which the NOPR had been collecting data under Medicare coverage with evidence development for nearly 7 years. These studies, beyond those originally reported by the NOPR in 2015 and 2016 (23-25), were designed to address criticisms of the existing data by the Centers for Medicare and Medicaid Services (CMS) that evidence of impact on appropriate management delivered to patients and on patient outcomes was lacking (26). Under newer CMS coverage policy, PET with ${ }^{68} \mathrm{Ga}$-PSMA-11 will be reimbursed for its labeled indication once this radiopharmaceutical is approved by the Food and Drug Administration, but that will provide no assurance of coverage by private insurers. Accordingly, the paper by Calais et al. (22) was aimed at proactively addressing this potential coverage disparity between Medicare and other third-party payers, thereby avoiding coverage issues encountered to date in the United States with ${ }^{68} \mathrm{Ga}$-DOTATATE and ${ }^{18} \mathrm{~F}$-fluciclovine. In all 3 studies, a large number of cases were analyzed within the NOPR frame- work, and changes in management plan were related to subsequent documentation of use of medical services and patient survival. Although all these papers warrant careful reading given the rich detail provided regarding the impact of these investigations on patient management, use of other health-care resources, and prognostic stratification, to my mind at least, the results are entirely predictable given the already proven accuracy of these techniques compared with prior diagnostic imaging paradigms. They speak more powerfully of the illogicality of the HTA process, which seeks to conflate diagnostic performance with outcomes that are multifactorial and only partially dependent of imaging results.

As detailed above, an imaging test is integrated into an already complex algorithm of diagnostic filtering that defines and refines the purpose of the investigation and affects both the pretest and the postlikelihood of disease. By more accurately defining the presence, extent, and nature of disease, a superior test will suggest that alternative management options might need to be used. However, the eventual outcome of patients will depend on whether clinicians or patients themselves choose to act on this information, the efficacy treatments available, and importantly, the natural history of the disease itself. We see the impact of these factors in the studies mentioned. In the report of Calais et al. (22), we see that despite the findings on ${ }^{68} \mathrm{Ga}-\mathrm{PSMA}-11$ leading to a change in management intention, a significant proportion of these decisions were not implemented. This is perhaps to be expected with a relatively new test that has substantially higher sensitivity than traditional imaging techniques (27). In a highly multidisciplinary clinical environment in which there are several and evolving therapeutic options (28), it may take time for the rationale integration of PSMA PET findings into treatment decision making. When we first introduced ${ }^{18} \mathrm{~F}$-FDG PET, there was also a significant rate of "no-impact" cases in which clinicians chose to ignore the PET findings. This generally involved patients being given the benefit of the doubt when the detection of suspected metastatic disease could not be independently verified by conventional investigations, as is not uncommon when transitioning to a more sensitive test. With time, physician confidence in PET increased as experience confirmed that such patients almost invariably had poor outcomes due to progression at these and other sites of metastasis despite a futile attempt at cure. As a result, no-impact studies diminished in later series. Judging PET by the initial failure of referring clinicians to appropriately integrate findings into management planning would have provided an unfair assessment of the utility of ${ }^{18} \mathrm{~F}$-FDG PET in earlier studies and yet this is what HTA methodologists would have us believe is a more appropriate surrogate for a test's utility than intended management impact (29). They further argue that randomized control trials that incorporate actual management delivered should be performed to determine the impact of diagnostic tests on patient outcomes (30). The equipoise involved in doing a trial in which patients are denied access to what has already been shown to be a more accurate test is questionable, at least in my mind. It is also incongruous that the intention-to-treat methodology applied in most randomized control trial designs is considered inappropriate when judging the impact of PET. In 1 early randomized control trial of ${ }^{18}$ F-FDG PET in lung cancer (31), several adverse outcomes in the PET arm came from clinicians ignoring the finding of metastatic disease and attempting curative treatment, and patients who did not even get the PET that they were randomized to have were included in the analysis of PET outcomes.

Although treatment decisions are much less likely to be controversial for ${ }^{18} \mathrm{~F}$-fluoride PET bone scans, since treatment 
algorithms for bone metastases are generally better established, there was a lower rate of concordance between intended treatment plans and inferred treatment in prostate cancer than in lung cancer (20), again probably reflecting the multidisciplinary nature of decision making and the rapidly evolving therapeutic landscape in this disease. Further, the paper of Gareen et al. (21) underlines the importance of disease biology in determining outcomes of patients after imaging, with the likelihoods of hospice admission or death within the reference follow-up period being substantially higher for patients with lung cancer than for those with prostate cancer. This likely reflects a combination of more effective treatments being available for bony metastatic prostate cancer and its more indolent natural history than is the case for lung cancer.

Although one must admire the effort that has gone into the advocacy for PET in response to criticisms of CMS and other HTA agencies, one can only lament how much time and potentially how many lives have been lost while patients have been denied access to what are clearly highly effective tests. Although the focus is importantly on use of medical resources and survival, we should also not forget the huge patient and societal advantages of not treating patients who are shown not to have disease with sufficient confidence to allow an observational or conservative approach. The technical and societal advantages of PET are compelling, but unreasonable regulatory hurdles pose almost insurmountable challenges to it replacing clearly inferior tests (32).

"Human beings, who are almost unique in having the ability to learn from the experience of others, are also remarkable for their apparent disinclination to do so."

—Douglas Adams, British writer, humorist, and dramatist

\section{DISCLOSURE}

Professor Hicks is a recipient of a National Health and Medical Research Council Practitioner Fellowship (APP1108050). No other potential conflict of interest relevant to this article was reported.

\section{REFERENCES}

1. Sullivan R, Peppercorn J, Sikora K, et al. Delivering affordable cancer care in high-income countries. Lancet Oncol. 2011;12:933-980.

2. Gambhir SS, Czernin J, Schwimmer J, Silverman DH, Coleman RE, Phelps ME. A tabulated summary of the FDG PET literature. J Nucl Med. 2001;42:1S-93S.

3. Fryback DG, Thornbury JR. The efficacy of diagnostic imaging. Med Decis Making. 1991;11:88-94.

4. Valk PE, Pounds TR, Tesar RD, Hopkins DM, Haseman MK. Cost-effectiveness of PET imaging in clinical oncology. Nucl Med Biol. 1996;23:737-743.

5. Hicks RJ, Kalff V, MacManus MP, et al. ${ }^{18}$ F-FDG PET provides high-impact and powerful prognostic stratification in staging newly diagnosed non-small cell lung cancer. J Nucl Med. 2001;42:1596-1604.

6. Mac Manus MP, Hicks RJ, Ball DL, et al. F-18 fluorodeoxyglucose positron emission tomography staging in radical radiotherapy candidates with nonsmall cell lung carcinoma: powerful correlation with survival and high impact on treatment. Cancer. 2001;92:886-895.

7. Hicks RJ, Kalff V, MacManus MP, et al. The utility of ${ }^{18}$ F-FDG PET for suspected recurrent non-small cell lung cancer after potentially curative therapy: impact on management and prognostic stratification. J Nucl Med. 2001;42:16051613 .

8. Kalff V, Hicks RJ, Ware RE, Hogg A, Binns D, McKenzie AF. The clinical impact of ${ }^{18}$ F-FDG PET in patients with suspected or confirmed recurrence of colorectal cancer: a prospective study. J Nucl Med. 2002;43:492-499.
9. Ware RE, Matthews JP, Hicks RJ, et al. Usefulness of fluorine-18 fluorodeoxyglucose positron emission tomography in patients with a residual structural abnormality after definitive treatment for squamous cell carcinoma of the head and neck. Head Neck. 2004;26:1008-1017.

10. Porceddu SV, Jarmolowski E, Hicks RJ, et al. Utility of positron emission tomography for the detection of disease in residual neck nodes after (chemo) radiotherapy in head and neck cancer. Head Neck. 2005;27:175-181.

11. Duong CP, Demitriou H, Weih L, et al. Significant clinical impact and prognostic stratification provided by FDG-PET in the staging of oesophageal cancer. Eur J Nucl Med Mol Imaging. 2006;33:759-769.

12. Duong CP, Hicks RJ, Weih L, et al. FDG-PET status following chemoradiotherapy provides high management impact and powerful prognostic stratification in oesophageal cancer. Eur J Nucl Med Mol Imaging. 2006;33:770-778.

13. Højgaard L. Are health technology assessments a reliable tool in the analysis of the clinical value of PET in oncology? Who audits the auditors? Eur J Nucl Med Mol Imaging. 2003;30:637-641.

14. Hicks RJ. Editorial: health technology assessment and cancer imaging: who should be setting the agenda? Cancer Imaging. 2004;4:58.

15. Ware R. Health technology assessment (HTA) groups have been instrumental in restricting patient access to PET worldwide. J Nucl Med. 2004;45:1977.

16. McEwan AJ. Does health technology assessment put patient care at risk? J Nucl Med. 2005;46:1939.

17. Ware RE, Hicks RJ. Doing more harm than good? Do systematic reviews of PET by health technology assessment agencies provide an appraisal of the evidence that is closer to the truth than the primary data supporting its use? J Nucl Med. 2011;52(suppl 2):64S-73S.

18. Hillner BE, Liu D, Coleman RE, et al. The National Oncologic PET Registry (NOPR): design and analysis plan. J Nucl Med. 2007;48:1901-1908.

19. Hillner BE, Siegel BA, Shields AF, et al. The impact of positron emission tomography (PET) on expected management during cancer treatment: findings of the National Oncologic PET Registry. Cancer. 2009;115:410-418.

20. Hillner BE, Hanna L, Makineni R, et al. Intended versus inferred treatment after ${ }^{18} \mathrm{~F}$-fluoride PET performed for evaluation of osseous metastatic disease in the National Oncologic PET Registry. J Nucl Med. 2018;59:421-426.

21. Gareen IF, Hillner BE, Hanna L, et al. Hospice admission and survival after ${ }^{18} \mathrm{~F}-$ fluoride PET performed for evaluation of osseous metastatic disease in the National Oncologic PET Registry. J Nucl Med. 2018;59:427-433.

22. Calais J, Fendler WP, Eiber M, et al. Impact of ${ }^{68} \mathrm{Ga}$-PSMA-11 PET/CT on the management of prostate cancer patients with biochemical recurrence. $J$ Nucl Med. 2018;59:434-441.

23. Hillner BE, Siegel BA, Hanna L, Duan F, Shields AF, Coleman RE. Impact of ${ }^{18} \mathrm{~F}$-fluoride PET in patients with known prostate cancer: initial results from the National Oncologic PET Registry. J Nucl Med. 2014;55:574-581.

24. Hillner BE, Siegel BA, Hanna L, et al. Impact of ${ }^{18} \mathrm{~F}$-fluoride PET on intended management of patients with cancers other than prostate cancer: results from the National Oncologic PET Registry. J Nucl Med. 2014;55:1054-1061.

25. Hillner BE, Siegel BA, Hanna L, Duan F, Quinn B, Shields AF. ${ }^{18}$ F-fluoride PET used for treatment monitoring of systemic cancer therapy: results from the National Oncologic PET Registry. J Nucl Med. 2015;56:222-228.

26. Decision memo for positron emission tomography (NaF-18) to identify bone metastasis of cancer (CAG-00065R2). Centers for Medicare and Medicaid Services website. https://www.cms.gov/medicare-coverage-database/details/ncadecision-memo.aspx?NCAId=279. Accessed January 9, 2018.

27. Perera M, Papa N, Christidis D, et al. Sensitivity, specificity, and predictors of positive ${ }^{68} \mathrm{Ga}$-prostate-specific membrane antigen positron emission tomography in advanced prostate cancer: a systematic review and meta-analysis. Eur Urol. 2016;70:926-937.

28. Hamid AA, Sweeney CJ. Prostate cancer: a new standard-of-care for advancedstage disease. Nat Rev Clin Oncol. 2017;14:592-593.

29. Kemp R, Prasad V. Surrogate endpoints in oncology: when are they acceptable for regulatory and clinical decisions, and are they currently overused? BMC Med. 2017; 15:134.

30. Staub LP, Lord SJ, Simes RJ, et al. Using patient management as a surrogate for patient health outcomes in diagnostic test evaluation. BMC Med Res Methodol. 2012;12:12.

31. Viney RC, Boyer MJ, King MT, et al. Randomized controlled trial of the role of positron emission tomography in the management of stage I and II non-smallcell lung cancer. $J$ Clin Oncol. 2004;22:2357-2362.

32. Hicks RJ, Hofman MS. Is there still a role for SPECT-CT in oncology in the PET-CT era? Nat Rev Clin Oncol. 2012;9:712-720. 